\title{
Mm-VLBI Phase Correction Capability with the Korean VLBI Network
}

\section{Taehyun Jung* and Bong Won Sohn}

Korea Astronomy and Space Science Institute

776 Daedeokdae-ro, Yuseong-gu, Daejeon, South Korea

University of Science and Technology

Gajeong-ro, Yuseong-gu, Daejeon, South Korea

E-mail: thjung@kasi.re.kr, bwsohn@kasi.re.kr

\section{Do-Young Byun}

Korea Astronomy and Space Science Institute

776 Daedeokdae-ro, Yuseong-gu, Daejeon, South Korea

E-mail: bdy@kasi.re.kr

\begin{abstract}
We present the results of VLBI phase correction demonstrated by the unique multi-frequency receiver system of Korean VLBI Network $(\mathrm{KVN})$ that can observe four radio bands (22/43/86/129 $\mathrm{GHz}$ ) simultaneously. In order to investigate the performance of the calibration of atmospheric phase fluctuations using this system we have conducted a simultaneous 4-frequency observation for 17 bright AGNs. The frequency phase transfer (FPT) that uses lower frequency phase (22 $\mathrm{GHz})$ to calibrate higher ones $(43 / 86 / 129 \mathrm{GHz})$ is applied and then the phase coherences at higher frequencies are significantly improved. These preliminary results are promising, and indicate potential offered by multi-frequency receiver system for mm-VLBI phase calibration and open new perspectives in the multi-frequency study of VLBI.
\end{abstract}

12th European VLBI Network Symposium and Users Meeting,

7-10 October 2014

Cagliari, Italy

\footnotetext{
* Speaker.
} 


\section{Introduction}

The mm-VLBI has a significant advantage in angular resolution which provides a powerful tool to study the fine structures of bright radio sources. However the sensitivity and imaging capability of radio interferometers are strictly limited by the atmospheric phase fluctuations [1]. VLBI phase referencing techniques which use the phase of calibrators to correct the phase of the target have been developed and applied to overcome these effects but they are not very successful at mmVLBI due to lower flux densities of radio sources, a higher system noise temperature and faster rotation of the fringe phase $[2,3]$.

Sasao [3] suggested the use of multi-frequency feeds as a tool for phase compensation in millimeter wavelength VLBI referencing. The troposphere is a non-dispersive medium; that is, the variation of excess path length is constant independent of the observing wavelength. Hence, the lower frequency fringe phases which are more stable with higher SNRs can be used for compensating the phases at higher frequencies. In multi-frequency feed system, target and reference sources are exactly same so as not to cause the loss of coherence due to the separation angle between them and also no reference source problem compared to other phase referencing methods [4].

\section{Simultaneous 4-frequency observation with KVN}

Based on those prospects in mm-VLBI, the Korean VLBI Network (KVN) introduced a multifrequency receiver system $(22 / 43 / 86 / 129 \mathrm{GHz})$ and its first simultaneous 4-frequency VLBI fringes on all KVN baselines were successfully obtained in 2012 [5]. After the first feasibility test of multifrequency receiver system, the simultaneous 4-frequency observation was performed to demonstrate the performance of mm-VLBI phase correction of KVN[4, 5]. We selected a total of 17 bright active galactic nuclei (AGNs) and each source was observed for an hour continuously in turn during the 24-hour observing experiment (see Fig. 1). A conventional phase referencing (fast antenna switching) scans were also conducted for the source pairs of 3C345 and NRAO512 for 30 minutes described as FAS(fast antenna switching) in (Fig. 1).

The data reduction was carried out with the AIPS(Astronomical Image Processing System) and no failed fringe solutions were found at $22 \mathrm{GHz}$ with the integration of 18 seconds (solint 0.3). Detected fringe SNRs have a range from 17 to 700 at $22 \mathrm{GHz}$. In order to calibrate high frequency VLBI phases (43/86/129 GHz), phase solutions obtained at $22 \mathrm{GHz}$ were copied to the high frequency data and then were updated by multiplying the frequency ratios $\left(v_{\text {high }} / v_{\text {low }}\right)$ of 2.0, 3.0, and 6.0 for 43, 86, $129 \mathrm{GHz}$, respectively. After applying the frequency phase transfer (FPT) that uses lower frequency phase solutions to calibrate higher frequency phase, visibility phases of higher frequency data are presented in the upper three plots in Fig. 1. Further details on the FPT method can be found in [4, 6]. For a comparison, the visibility phases at $86 \mathrm{GHz}$ calibrated by using $43 \mathrm{GHz}$ phase solutions are also shown in the bottom plot of Fig. 1.

\section{Results and Discussions}

As shown in Fig. 1, the results of FPT imply that simultaneous multi-frequency observations 


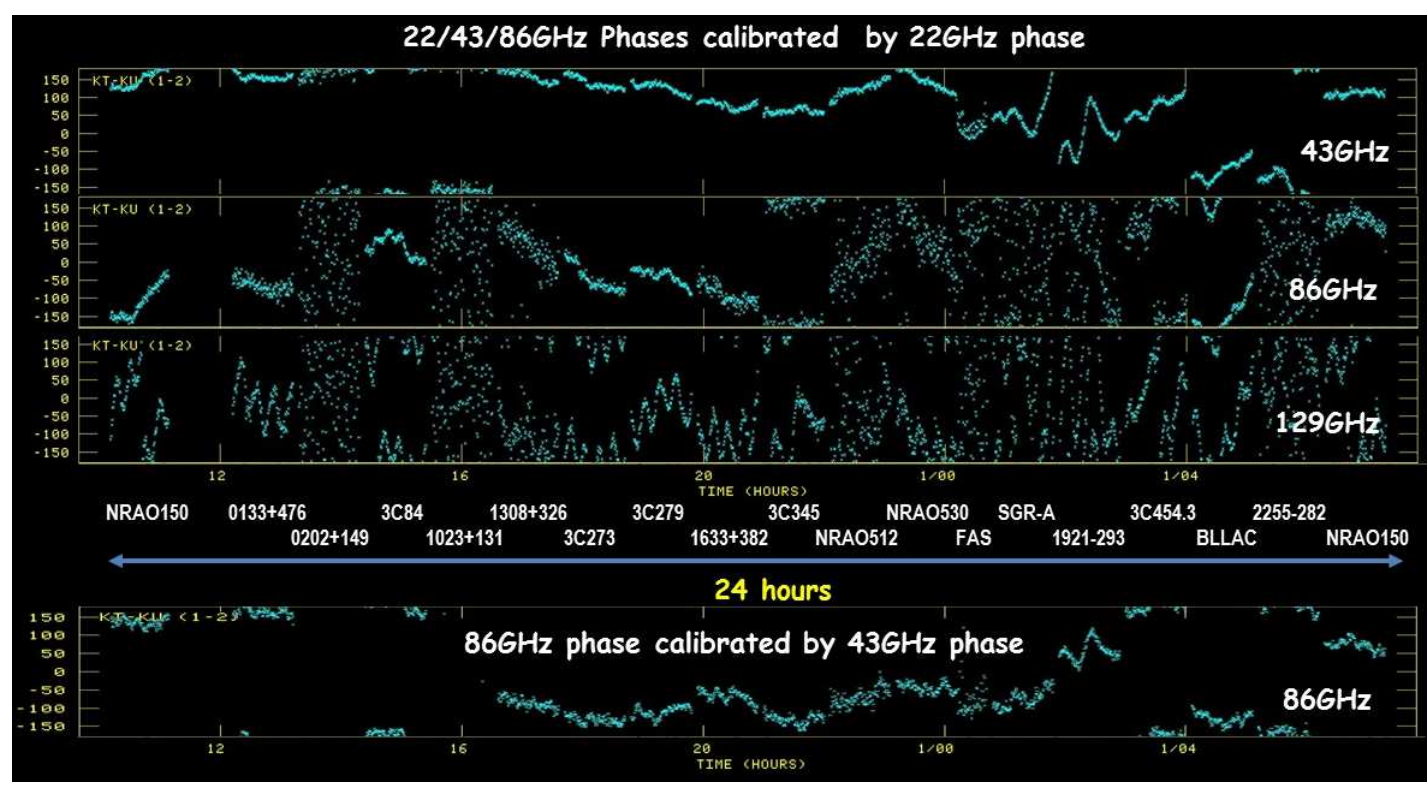

Figure 1: (Top) Visibility phases at 43/86/129 GHz calibrated by using the $22 \mathrm{GHz}$ phase solutions. (Bottom) Visibility phases at $86 \mathrm{GHz}$ calibrated by using the $43 \mathrm{GHz}$ phase solutions. Each source was observed for an hour continuously in turn during the 24-hour observing experiment at the KVN Tamna and Ulsan baseline.

can effectively compensate for atmospheric phase fluctuations in mm-VLBI . Visibility phases at 43, 86, $129 \mathrm{GHz}$ are considerably stabilized by FPT, showing a smooth long term phase variation for a day. The results of phase correction at 86 and $129 \mathrm{GHz}$ are worse than those at $43 \mathrm{GHz}$ because the phase solution errors from $22 \mathrm{GHzdata}$ are transferred to the higher frequencies multiplied by frequency ratios $\left(v_{\text {high }} / v_{\text {low }}\right)$ that become larger. As an example, the phase corrections failed at 86 and $129 \mathrm{GHz}$ for the sources of $0202+149$ and 1023+131 where sources have relatively lower SNRs (17 23) at $22 \mathrm{GHz}$ fringe solutions (solint 0.3) and for lower elevation sources such as Sgr A and 2255-282. We found that the sinusoidal patterns at $129 \mathrm{GHz}$ are caused by temperature variation of the receiver cabin. The comparison of phase correction results at $86 \mathrm{GHz}$ between FPT from 22 $\mathrm{GHz}$ and $86 \mathrm{GHz}$ clearly shows the error propagation effects. If the source is detectable at $43 \mathrm{GHz}$ with enough SNRs the FPT results at $86 \mathrm{GHz}$ calibrated by $43 \mathrm{GHz}$ are better than $22 \mathrm{GHz}$. Thus, the reference frequency for FPT is selected by taking into the significance of the detection (SNR) and frequency ratios. These preliminary results are promising, and indicate potential offered by multi-frequency receiver system for mm-VLBI phase calibration and open new perspectives in the multi-frequency study of VLBI.

\section{References}

[1] A. E. E. Rogers et al., 1984, Radio Sci., 19, 1552

[2] J. M. Moran \& B. R. Rosen, 1981, Radio Sci., 16, 235

[3] T. Sasao, 2003, ASP Conf. Ser., 306, 53

[4] T. Jung et al., 2011, PASJ, 63, 375 
[5] T. Jung et al., in proceedings of 11th EVN Symposium, PoS (EVN 2012) 060 (2012)

[6] M. J. Rioja et al., in proceedings of 12th EVN Symposium, PoS (EVN 2014 ) 047 (2014) 